\title{
A conservation law model for bidensity suspensions on an incline
}

\author{
Jeffrey T. Wong, Andrea L. Bertozzi \\ Department of Mathematics, University of California, Los Angeles, \\ 520 Portola Plaza, Box 951555, Los Angeles, CA 90095-1555, USA
}

\begin{abstract}
We study bidensity suspensions of a viscous fluid on an incline. The particles migrate within the fluid due to a combination of gravity-induced settling and shear induced migration. We propose an extension a recent model [N. Murisic, B. Pausader, D. Peschka, and A. L. Bertozzi, Dynamics of particle settling and resuspension in viscous liquid films, Journal of Fluid Mechanics 717 (2013), 203-231] for monodisperse suspensions to two species of particles, resulting in a hyperbolic system of three conservation laws for the height and particle concentrations. We analyze the Riemann problem and show that the system exhibits three-shock solutions representing distinct fronts of particles and liquid traveling at different speeds as well as singular shock solutions for sufficiently large concentrations, for which the mechanism is essentially the same as the single-species case. We also consider initial conditions describing a fixed volume of fluid, where solutions are rarefaction-shock pairs, and present a comparison to recent experimental results. The long-time behavior of solutions is identified for settled mono- and bidisperse suspensions and some leading-order asymptotics are derived in the single-species case for moderate concentrations.
\end{abstract}

Keywords: Riemann Problem, bidensity slurry, conservation law

\section{Introduction}

Non-colloidal suspensions of particles in a shear flow exhibit complex interactions within the mixture. These suspensions have many applications, for example in modeling spiral separators used in the mining industry 7]. For suspensions on an incline, the competing effects of settling due to gravity and shear-induced migration lead to an interesting phenomenon [17, 1]: for low concentrations, there is a 'settled' regime in which the particles and fluid separate into distinct fronts, while for sufficiently high concentrations there is a 'ridged' regime in which only a single particle-rich front appears. Murisic et. al. [12, 13] developed a model for monodisperse suspensions on an incline based on the diffusive flux model of Acrivos [9, 16, which was successfully used to predict the experimentally observed settled and ridged regimes and the time evolution of the fronts. In this model the particles are assumed to be in equilibrium in the normal direction and the leading order system for the film height and particle concentrations form a hyperbolic system of conservation laws with fluxes determined by a system of ODEs that describe the distribution of particles in the normal direction. Analysis of hyperbolic systems arising for bidisperse settling have been extensively studied in the context of other models, for instance in the case of settling in a quiescent fluid [2], though not in the incline geometry where we are interested

Email addresses: jtwong@math.ucla.edu (Jeffrey T. Wong), bertozzi@math.ucla.edu (Andrea L. Bertozzi) in the long-term dynamics of the fronts rather than the settling of particles to the substrate. The Riemann problem has been studied for the monodisperse model [10, 20], showing the existence of double shock solutions in the settled regime and a transition to singular shocks that occurs in the ridged regime, where particles accumulate at the front. The corresponding rarefaction-singular shock solutions that arise for constant volume initial conditions have also been studied [21.

In this work, we propose an extension of the model to bidisperse suspensions by employing a modification of the diffusive flux model to multiple species [18, 19] and present some preliminary with comparison to recent experiments that build on previous work to identify the qualitative behavior of the fronts in the incline problem 6]. In section 2 . we present the model and discuss the details of the particle fluxes. In section section 3 we describe the Riemann problem for the system. The essential difference from the single species problem is the presence of an additional trailing shock delimiting the transition from heavier to lighter particles, while the remaining two shocks have a similar structure to their monodisperse counterparts. Due to the increased complexity of the system, it is more difficult to obtain analytical results, so we describe the system qualitatively and through numerical simulations. In section section 4, we consider fixed-volume solutions corresponding to results from experiments in which the particles settle to the substrate. We note that the Riemann problem is still relevant here to describe local shock solutions. The full solution has the form of a sequence of rarefaction-shock 
pairs corresponding to different fronts for the particles and fluid. We derive theoretical results for the long-time behavior of monodisperse suspensions in the settled regime, where the concentration of particles uniformly approaches a critical concentration independent of initial conditions. The asymptotic behavior of the front positions is derived, extending the existing results for the high-concentration [21, 20] and dilute limit [13. Then, some results are then extended to the bidisperse problem. Finally, in section 5 . we present a qualitative comparison of the front positions to recent experiments for the bidisperse problem.

\section{Model}

Our goal is to derive a system of hyperbolic conservation laws governing the evolution of the film height and particle concentrations for a bidensity suspension. This follows the standard lubrication approach for thin viscous films, but is complicated by the dependence of the velocity profiles on the particle distributions. Our model is an extension of the dynamic model proposed by Murisic et. al. 13] to multiple species of particles, making use of the equilibrium theory for the diffusive flux model for bidisperse suspensions [19, 5]. We consider a mixture of a fluid with large viscosity $\mu_{\ell}$ and density $\rho_{\ell}$ and two species of negatively buoyant particles with diameter $d$ and densities $\rho_{p, 1}, \rho_{p, 2}$ satisfying $\rho_{\ell}<\rho_{p, 1}<\rho_{p, 2}$. The suspension is allowed to spread down an incline of angle $\alpha$. The geometry of the system is summarized in Figure 1; we restrict our attention to the two-dimensional problem where the fluid does not vary in the span-wise direction of the incline. The mixture is assumed to be Newtonian with an effective viscosity $\mu(\phi)$ that depends on the particle concentration $\phi$ and density $\rho(\phi)=(1-\phi) \rho_{\ell}+\phi_{1} \rho_{p, 1}+\phi_{2} \rho_{p, 2}$. The equations for suspension momentum and particle mass conservation are

$$
\begin{gathered}
\rho \frac{\partial \boldsymbol{u}}{\partial t}+\rho \boldsymbol{u} \cdot \nabla \boldsymbol{u}=\nabla \cdot\left(-p I+\mu\left(\nabla \boldsymbol{u}+\nabla \boldsymbol{u}^{T}\right)\right)+\rho \mathbf{g} \\
0=\frac{\partial \phi_{i}}{\partial t}+\boldsymbol{u} \cdot \nabla \phi_{i}+\nabla \cdot \boldsymbol{J}_{i}, \quad i=1,2 .
\end{gathered}
$$

with $\boldsymbol{u}=(u, w)$. The mixture viscosity is given by the Krieger-Dougherty relation $\mu(\phi)=\mu_{\ell}\left(1-\phi / \phi_{\max }\right)^{-2}$.

The derivation of the conservation law model uses the standard thin-film approximation to reduce (1) to an equation for the film height and is summarized here, following [13. The equations are non-dimensionalized according to the scales

$$
\begin{gathered}
(\hat{x}, \hat{z})=H(x / \epsilon, z), \quad(\hat{u}, \hat{w})=\frac{H^{2} \rho_{\ell} g \sin \alpha}{\mu_{\ell}}(u, \epsilon w), \\
\hat{p}=\frac{U \mu_{\ell}}{H} p, \quad\left(\hat{J}^{(x)}, \hat{J}^{(z)}\right)=\frac{d^{2} U}{H^{2}}\left(\epsilon J^{(x)}, J^{(z)}\right)
\end{gathered}
$$

where $U=H^{2} \rho_{\ell} g \sin \alpha / \mu_{\ell}$ and $\epsilon \ll 1$. Hereafter, all variables are non-dimensionalized and the hats are dropped for brevity. To simplify the model, we now make the assumption that the particles equilibrate quickly in the normal direction compared to the fluid flow down the incline, which requires that $\epsilon \ll(d / H)^{2} \ll 1$. The separation of time scales allows the dynamics in the $z$ and $x$-directions to be considered separately. In the $z$-direction, the particles are always in equilibrium, i.e.

$$
\boldsymbol{J}_{i}^{(z)}=0, \quad i=1,2 .
$$

To leading order in $\epsilon$, the non-dimensionalized $x$-component of (1) becomes

$$
\left(\mu u_{z}\right)_{z}=-\rho
$$

We impose the no-slip boundary condition on the incline surface and stress-balance on the free surface:

$$
\left.u\right|_{z=0}=0,\left.\quad \mu u_{z}\right|_{z=h}=0 .
$$

We want to integrate in $z$ to obtain equations for the film height $h(x, t)$ and depth-averaged concentrations

$$
\phi_{0, i}(x, t)=\frac{1}{h} \int_{0}^{h} \phi_{i}\left(x, z^{\prime}, t\right) \mathrm{d} z^{\prime} .
$$

It will also be convenient to define $\phi_{0}=\phi_{0,1}+\phi_{0,2}$ and $X_{0}=\phi_{0,1} / \phi_{0}$. Conservation of mass for the fluid and particles, to leading order, is the system

$$
\begin{aligned}
& 0=\frac{\partial h}{\partial t}+\frac{\partial}{\partial x}\left(\int_{0}^{h} u \mathrm{~d} z\right), \\
& 0=\frac{\partial\left(h \phi_{0, i}\right)}{\partial t}+\frac{\partial}{\partial x}\left(\int_{0}^{h} u \phi_{i} \mathrm{~d} z\right), \quad i=1,2 .
\end{aligned}
$$

It remains to find the $x$-velocity profile $u(x, z, t)$. For a clear fluid, this would be computed explicitly by integrating (4) with the boundary conditions (5), but the dependence on the concentration $\phi$ (through $\rho$ ) means that (3) must also be used to determine the particle distribution in $z$. Equations (3) and (4) form a system of ordinary differential equations in $z$ for the particle concentration $\phi$, proportion of lighter particles $X=\phi_{1} / \phi$ and shear stress $\sigma=\mu(\phi) \frac{\partial u}{\partial z}$. To remove the dependence on the film height $h(x, t)$ we use the scaled variable $s=z / h$ and scaled quantities $\widetilde{\sigma}(x, s, t)=h^{-1} \sigma(x, h s, t), \widetilde{\phi}(x, s, t)=\phi(x, s h, t)$ and $x$-velocity $\widetilde{u}(x, s, t)=h^{-2} u(x, s h, t)$.

By rewriting the integrals in terms of $s=z / h$ and using the solution to the equilibrium system 10 , we can write the system (7) in the more useful form

$$
\begin{aligned}
& 0=\frac{\partial h}{\partial t}+\frac{\partial}{\partial x}\left(h^{3} f\left(\phi_{0}, X_{0}\right)\right), \\
& 0=\frac{\partial\left(h \phi_{0, i}\right)}{\partial t}+\frac{\partial}{\partial x}\left(h^{3} g_{i}\left(\phi_{0}, X_{0}\right)\right) . \quad i=1,2 .
\end{aligned}
$$

The functions $f, g$ are obtained by integrating the scaled velocity profile $\widetilde{u}$ :

$$
f\left(\phi_{0}, X_{0}\right)=\int_{0}^{1} \tilde{u} \mathrm{~d} s, \quad g_{i}\left(\phi_{0}, X_{0}\right)=\int_{0}^{1} \tilde{u} \tilde{\phi}_{i} \mathrm{~d} s .
$$


Note that adding the two equations in $8 \mathrm{~b}$ gives a pair of conservation laws for the film height and total number of particles, similar to the monodisperse problem [13. However, the system is not closed because $g$ depends on the concentration of both species, so the third equation is necessary. Nevertheless, the reduced system for $h$ and $\phi_{0}$ informs much of the structure of the full problem, which allows the existing theory to be applied to the bidisperse case.

To close the system, we specify the model for the particle fluxes and obtain the particle ODE. Following the diffusive flux model of Acrivos [8, 16] and an extension to multiple species [19], the flux is a sum of contributions due to polydisperse settling [18, shear-induced diffusion and mixing of particles:

$$
\boldsymbol{J}_{i}=\boldsymbol{J}_{\text {settling }, i}+\boldsymbol{J}_{\text {shear }}+\boldsymbol{J}_{\text {tracer }, i} .
$$

The shear-induced migration term is

$$
\boldsymbol{J}_{\text {shear }}=-\frac{d^{2} K_{c} \phi}{4} \nabla(\dot{\gamma} \phi)-\frac{d^{2}}{4} \frac{K_{v} \dot{\gamma} \phi^{2}}{\mu(\phi)} \nabla \mu(\phi)
$$

where $d$ is the particle diameter, $\dot{\gamma}=$ is the shear rate. and the constants $K_{c} \approx 0.41$ and $K_{v} \approx 0.62$ are empirically determined [16]. The flux due to settling for multiple species is

$$
\boldsymbol{J}_{\text {settling }, i}=\frac{d^{2} \mathbf{g}}{18 \mu_{\ell}}\left(M_{0}\left(\rho_{i}-\rho_{\ell}\right)+M_{I} \sum_{j=1}^{2}\left(\rho_{p, j}-\rho_{\ell}\right) \frac{\phi_{i}}{\phi}\right),
$$

where $M_{0}=1-\frac{\phi}{\phi_{m}}$ and $M_{I}=\Phi(\phi)$ are the self- and interaction mobilities [18, 19. Lastly, to account for mixing between particle species due to shear-induced migration, we include a flux $J_{\text {tracer }}$ given by

$$
\boldsymbol{J}_{\text {tracer }, i}=-\frac{\dot{\gamma} d^{2}}{4} D_{\mathrm{tr}}(\phi) \phi \nabla\left(\frac{\phi_{i}}{\phi}\right) .
$$

Equations (3) and (4) form a system of ordinary differential equations in $z$ for the particle concentration $\phi$, proportion of lighter particles $X=\phi_{1} / \phi$ and shear stress $\sigma=\mu(\phi) \frac{\partial u}{\partial z}$. The result is the system

$$
\begin{aligned}
\frac{\partial \widetilde{\sigma}}{\partial s} & =-1-\widetilde{\phi} \rho(\widetilde{X}), \\
\frac{\partial \widetilde{X}}{\partial s} & =c_{2} \frac{1}{\sigma} \frac{1}{D_{t r}(\widetilde{\phi})} \widetilde{X}(1-\widetilde{X}) \frac{\phi_{\max }}{\phi_{\max }-\widetilde{\phi}}, \\
\frac{\partial \widetilde{\phi}}{\partial s} & =\frac{\left(\phi_{\max }-\widetilde{\phi}\right)\left(\widetilde{\phi}+\rho(\widetilde{X})\left(\widetilde{\phi}^{2}-B(1-\widetilde{\phi})\right)\right)}{\sigma\left(\phi_{\max }-\widetilde{\phi}+c_{1} \widetilde{\phi}\right)} .
\end{aligned}
$$

with boundary condition $\widetilde{\sigma}(1)=0$ from $(5)$. Here $\rho(X)=$ $\rho_{s, 1} X+\rho_{s, 2}(1-X)$ is an average particle density, $B=$ $\frac{2 \cot \alpha}{9 K_{c}}$ describes the balance of gravity-induced settling and shear-induced migration and $c_{1}=2\left(K_{v} / K_{c}-1\right)$. The $x$ velocity profile is obtained from the shear stress as $\widetilde{u}(x, s, t)=$ $\int_{0}^{s} \mu\left(\widetilde{\phi}\left(x, s^{\prime}, t\right)\right)^{-1} \widetilde{\sigma}\left(x, s^{\prime}, t\right) \mathrm{d} s^{\prime}$, from which the fluxes 9 are computed.

Before analyzing solutions in the next section, we describe a few salient features of the fluxes. In the case of no particles, $f=1 / 3$ is a constant and $g_{i}=0$ in accordance with the well-studied clear-fluid model [3]. For sufficiently large concentrations the fluxes vanish, i.e. there is a maximum packing fraction $\phi_{m}$ such that $f\left(\phi_{m}\right)=g\left(\phi_{m}\right)=0$. In addition, if the inclination angle is not too small then for each $X$ there is a unique critical concentration $\phi_{c}=$ $\phi_{c}\left(X_{0}\right) \in\left(0, \phi_{m}\right)$ such that $\phi_{c} f\left(\phi_{c}, X_{0}\right)=g\left(\phi_{c}, X_{0}\right)$. We set $\phi_{c}$ to be this value if it exists and $\phi_{m}$ otherwise. This is analogous to the case of a single species [12, but the critical concentration now depends on the relative concentration of each particle type. As it appears frequently in the analysis, we define the quantity

$$
R\left(\phi_{0}, X_{0}\right)=\phi_{0} f\left(\phi_{0}, X_{0}\right)-g\left(\phi_{0}, X_{0}\right) .
$$

Physically, $R$ represents the difference between the difference in effective fluid and particle velocities, so if $R>0$ then the particles and fluid will tend to separate into distinct fronts and aggregate at a single front if $R<0$. Following the notation for the monodisperse theory [12, we call a state with some concentration $\phi_{0}$ and $X_{0}$ 'settled' if $\phi_{0}<\phi_{c}$ and 'ridged' if $\phi_{0}>\phi_{c}$, corresponding to the two regimes observed in experiments.

Plots of the fluxes $f$ (fluid) and $g_{i}$ (particle $i$ ), along with $g=g_{1}+g_{2}$, are shown in Figure 2 as functions of $\phi$ and $X$. Hereafter, unless otherwise noted, computed results refer to $\alpha=30 \mathrm{deg}$ with parameters corresponding to experiments as in [5]. A few properties of the fluxes are worth noting here. Most importantly, the fluid flux $f$ is insensitive to changes in $X$; it only varies a small amount up to moderate $\phi$ and is nearly constant in $X$ for when $\phi_{0}>\phi_{c}(0)$, the critical concentration for the heavier particles from the monodisperse theory [12. Similarly, the total particle flux $g$ is nearly constant in $X$ for $\phi_{0}>\phi_{c}(0)$ as well, although it varies considerably with $X$ below this threshold (as do the fluxes for each species).

For the fluxes of the equilibrium model, we can look at $\frac{\partial f}{\partial X}$ in more detail to try to understand why it tends to be small, although are more precise estimate is currently lacking. Consider a solution $(\tilde{\phi}, \tilde{X}, \tilde{\sigma})$ to 10 with a given total concentration $\phi$ and $X$. The density difference $\Delta \rho$ is not small, so one might expect there to be a significant dependence on $\rho$. According to the equilibrium theory (as a consequence of $(10 \mathrm{~b})$, the ODE for $\tilde{X})$, the particle layers are mostly stratified in equilibrium, so we can approximate

$$
\tilde{X}(s)= \begin{cases}0 & s<s_{t} \\ 1 & s>s_{t}\end{cases}
$$

for some transition point $s_{t}$ (past which there are no heavier particles). From 10a, the change in shear stress with $X$ is then

$$
\frac{d \tilde{\sigma}}{d X}(s)=\left\{\begin{array}{ll}
\left(\rho_{1}-\rho_{2}\right) \phi & s<s_{t} \\
0 & s>s_{t}
\end{array} .\right.
$$


The change in $f$ is given by the expression

$$
\begin{aligned}
\frac{\partial f}{\partial X}=-\Delta \rho \phi \int_{0}^{s_{t}} & (1-s)\left(1-\frac{\tilde{\phi}(s)}{\phi_{m}}\right)^{2} d s \\
& -\frac{2}{\phi_{m}} \int_{0}^{1}(1-s)\left(1-\frac{\tilde{\phi}}{\phi_{m}}\right) \sigma \frac{\partial \phi}{\partial X} \mathrm{~d} s
\end{aligned}
$$

The first term is due to the change in shear stress and is negative. The second term represents the change in $f$ due to the concentration profile change (which affects the viscosity). The sign of $\frac{\partial \phi}{\partial \tilde{X}}$ is negative for $s<s_{t}$ (an increase in the number of lighter particles will lead to a smaller total concentration near the substrate). While neither term is particularly small in general, they tend to oppose each other. In the ridged regime, where $\phi$ is increasing, we expect $\frac{\partial f}{\partial \tilde{X}}$ to be small (as is evident in Figure 2) because $\mu^{-1}$ and $d\left(\mu^{-1}\right) / d \phi$ will both be small by to the viscosity relation.

\section{Riemann Problem}

To understand the essential structure of solutions to the system (8), we first look at the Riemann problem. In [21, it was shown that for a single species, there are three types of solutions: in the settled regime, a pair of shocks; and in the ridged regime, either a pair of shocks with a large intermediate height or a singular shock where particles collect at the leading front. For the bidsperse system, the same is true; numerically computed profiles in each case are shown in Figure 3. The new feature is an additional, slower shock containing heavier particles which leads to a separation of the two species. In this section, we investigate this third shock and its effect on the solutions.

It is convenient to write the system in vector form, so we set $U=\left(h, h \phi_{0,1}, h \phi_{0,2}\right)^{T}$ and $F(U)=h^{3}\left(f, g_{1}, g_{2}\right)^{T}$ so that the system is

$$
\frac{\partial U}{\partial t}+\frac{\partial}{\partial x}(F(U))=0
$$

with Riemann initial conditions

$$
U(x, 0)= \begin{cases}U^{L} & x<0 \\ U^{R} & x>0\end{cases}
$$

(the superscripts indicate the state, e.g. $L$ for left state). Note that $F$ depends only on $h$ in the leading factor of $h^{3}$. While the system is defined in terms of the conserved variables $h \phi_{0, i}$ it will be more useful in computations to use the total/relative concentrations $\phi_{0}, X_{0}$, which are not conserved. The Jacobian of the system is

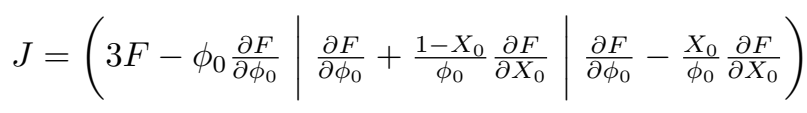

which has eigenvalues $\lambda_{k}$ satisfying $0<\lambda_{1}<\lambda_{2}<\lambda_{3}$. That the system is hyperbolic has been verified numerically over the relevant parameter regime by checking that the eigenvalues are in fact real and positive over all valid values of $\phi$ and $X$.

We summarize some of the relevant theory here, details of which can be found e.g. in 4. A general hyperbolic system of the form 12 with initial conditions 13 admits weak solutions in which the discontinuity with left/right values $U^{L}, U^{R}$ travels at a speed $s$ satisfying the RankineHugoniot condition

$$
F\left(U^{L}\right)-F\left(U^{R}\right)=s\left(U^{L}-U^{R}\right)
$$

For a system and a fixed left state $U^{L},(14)$ defines the Hugoniot locus, the set of right states for which a shock exists connecting $U^{L}$ to $U^{R}$. To ensure uniqueness, we impose the Lax entropy condition for the shock, which requires that

$$
\lambda_{k}\left(U^{L}\right)>s>\lambda_{k}\left(U^{R}\right), \lambda_{k+1}\left(U^{R}\right)>s>\lambda_{k-1}\left(U^{L}\right) .
$$

for some integer $k$. For each state $U$ the Hugoniot locus consists of a smooth curve through $U$ for each $k$ with two branches $S_{k}^{-}(U)$ and $S_{k}^{+}(U)$ that satisfy (14) and (15) with $U$ as left or right state, respectively.

In general, the system 12 with initial conditions (13) is not guaranteed to have a weak solution, but solutions can be constructed when they exist using the shock curves. Of interest here are triple shock solutions, that is, a sequence of shock connections $U^{L}=U^{(0)} \rightarrow U^{(1)} \rightarrow U^{(2)} \rightarrow$ $U^{(3)}=U^{R}$ with the $k$-shock $U^{(k-1)} \rightarrow U^{(k)}$ satisfying 15. Completely solving the Riemann problem also requires constructing rarefaction solutions; for now, we restrict our discussion to solutions only with shocks. Rarefactions are discussed in the context of constant volume solutions in section 4 .

\subsection{First shock}

We consider now in detail the 1-shock for the bidisperse system. Exact results for the solutions are somewhat difficult to obtain due not only to the equilibrium ODE, but also to the three-equation system, so we rely on numerically computed shock curves to construct the solution. A family of numerically computed shock curves $S_{1}^{-}$different left-state concentrations $\phi_{L}$ are shown in Figure 4. To a good approximation, the curve for small $\phi_{L}$ represents a jump only in $X_{0}$, with $h$ and $\phi_{0}$ constant. There is only a small variation in $h$ (representing a small downward jump in film height that can be seen in Figure 3). Note that the admissible states $U^{(1)}$ along the shock curve have $X_{0}>X_{L}$, an increase in the concentration of lighter particles. Physically, this represents the lighter particles and fluid separating from the slower front of heavier particles which lags behind while keeping the total concentration across the jump approximately constant. However, as evident in Figure 4, there is some variation in $h$, which is 
quite prominent as $\phi_{L}$ becomes large. When $X_{0} \neq 0$, the curve $S_{1}^{-}$will never be exactly parallel to the $X$ axis. This can be seen by noting that $U=(0,1,-1)$ in conserved variables points in the $X$-direction and is a right eigenvector of the Jacobian of 12 only if $\frac{\partial f}{\partial X_{0}}$ and $\frac{\partial g}{\partial X_{0}}$ are both zero (the case in which there is only one particle species). While $\frac{\partial f}{\partial X_{0}}$ is small (see Figure 2), the same is not true of the latter, so the 1-shock curve cannot only be a jump in $X_{0}$ and must have some change in $h$ or $\phi_{0}$.

Interestingly, the deviation in the $h\left(\right.$ and $\left.\phi_{0}\right)$ directions increases considerably near and beyond the critical concentration. This can be seen by assuming a formal expansion $h=h_{L}+h_{1}+\cdots, \phi_{0}=\phi_{L}+\cdots$ with $h_{1} \ll h_{L}$ where $h_{1} \ll h_{L}$ and the ellipses indicate higher order terms. In this case the $g_{1}$ equation in (14) gives an estimate for the shock speed:

$$
\frac{s}{h_{L}^{2}} \approx \frac{1}{\phi_{L}} \frac{g_{1}\left(\phi_{L}, X_{0}\right)-g_{1}\left(\phi_{L}, X_{L}\right)}{X_{0}-X_{L}} .
$$

Notably, $g_{1}$ is approximately quadratic in $X_{0}$, which implies that $s$ scales almost linearly with the proportion of lighter particles. Solving for $h_{1}$ gives the simple approximation

$$
\frac{h_{1}}{h_{L}} \approx \frac{1}{3} \frac{\left.\left(\phi_{0} f-g\right)\right|_{X_{L}} ^{X_{0}}}{\left.\left(\phi_{0} f-g\right)\right|_{X_{L}}}
$$

so $h_{1}$ depends on the jump in $\phi_{0} f-g$ from the left to right state. The effect can be seen in Figure 4 (plotting the 1curve for varying $\phi_{L}$ and fixed $X_{L}=0.5, h_{L}=1$ ). As $\phi_{L}$ comes close to the ridged regime, the shock curves begin to bend considerably in $h$ due to a large relative change in $\phi_{0} f-g$ (which is zero in the transition from settled to ridged).

\subsection{Second and third shocks}

Next, we consider the structure of the remaining two shocks. To construct a solution, we look at the surface of intermediate states $U^{(2)}$ that lie on 2-shock curves $S_{2}^{-}$emanating from states $U^{(1)} \in S_{1}^{-}\left(U^{L}\right)$. The desired solution is then obtained by intersecting the 3-curve $S_{3}^{+}\left(U^{R}\right)$ with this surface, which is shown in Figure 5 ). We also show the non-entropy half $S_{2}^{+}$of the 2-curves for later comparison with the ridged regime.

If the left state lies in the settled regime, $S_{2}^{-}$connects states $U^{(1)} \in S_{1}^{-} U_{L}$ to a second intermediate state with a smaller height and total concentration. If $\phi_{R}$ is small (or $X_{R}$ is large), i.e. there are few heavy particles downstream, then $X_{1}$ must be near one, so that the last intermediate state $U_{2}$ has $\phi_{0,2}$ sufficiently small (note that $\phi_{0,2} \rightarrow 0$ as $\left.X_{1} \rightarrow 1\right)$.

To complete the solution, we look at the 3-curve; we see that so long as $X_{R}$ is not too large, the 3-curve will indeed intersect the surface. From the $\left(h, \phi_{0}\right)$ projection (see right panel in Figure 5, it is clear that $\left(h_{R}, \phi_{R}\right)$ is required to be within a certain region, in agreement with the monodisperse theory [10]. If $\left(h_{R}, \phi_{R}\right)$ lies outside this region, then the 3 -connection is instead a rarefaction.
In the case of a ridged left state, the 2-curves satisfying Equation 15 instead have $h$ increasing rather than decreasing; the change in sign of $\phi_{0} f-g$ changes which branch of the 2-curve satisfies the entropy condition (this is illustrated in Figure 5, where both $S_{2}^{-}$and $S_{2}^{+}$are shown). The structure of the curves is somewhat more interesting (see [20]) and the asymptotic form is known in the limit as $\phi_{0} \rightarrow \phi_{m}$. The shock curves are shown in Figure 6. As $\phi$ increases, the value of $h_{L}$ increases and diverges to $\infty$, and for sufficiently large $\phi$, the 2 and $3-$ curves no longer intersect as $h \rightarrow \infty$, which leads to a singular shock. The monodisperse theory applies in this context because the curves in the bidisperse problem collapse almost exactly onto the $\left(h, \phi_{0}\right)$ plane. Again, as in the settled case, the 2-curves form a surface in the $\left(\phi_{0}, X_{0}\right)$ plane, so that the existence of an intersection $\left(h^{*}, \phi^{*}\right)$ in the $(h, \phi)$ plane implies there exists some state $U_{1}$ (by choosing the appropriate $X_{1}$ ) such that this is actually an intersection in the full three-dimensional system. Also, we can see that $X_{0} \rightarrow 1$ as $\phi \rightarrow \phi_{m}$ for the 2 and 3 -curves; this implies that only lighter particles aggregate at the singular 'front' (to be expected due to the increased tendency to settle of the heavier particles $[5$.

\section{Constant volume solutions}

It is of interest to study similarity solutions that arise as long time limits for these models when the initial condition is a reservoir of fluid of finite volume. This corresponds to the scenario studied in experiments. The simplest example is the Huppert solution for a clear fluid, which predicts a $t^{1 / 3}$ scaling for the front position [3. In the settled regime, there are multiple fronts - a leading front containing only clear fluid and slower front containg particles of each species. In subsection 4.1, we determine through an asymptotic analysis the long-term behavior of the particle front in the case of a single particle species. Then, in subsection 4.3 the results are extended to multiple species, and in section 5 a brief comparison to experiments is presented.

In our detailed analysis we look at the single-species case, in which (8) reduces to the system

$$
\begin{aligned}
& 0=h_{t}+\left(h^{3} f\left(\phi_{0}\right)\right)_{x} \\
& 0=\left(h \phi_{0}\right)_{t}+\left(h^{3} g\left(\phi_{0}\right)\right)_{x}
\end{aligned}
$$

for fluxes $f, g$ that depend only on $\phi_{0}$. To review the relevant theory (as in 4]), a hyperbolic system (8) has similarity solutions $U(x, t)=U(\xi)$ in the similarity variable $\xi=x / t$ which satisfy

$$
(J(U(\xi))-\xi I) U(\xi)=0,
$$

where $J(U)$ is the Jacobian of $F$. The solution $U(\xi)$ is an eigenvector correpsonding to an eigenvalue of the Jacobian $J$. If this eigenvalue is $\xi=\lambda_{k}(U(\xi))$ with corresponding eigenvector $r_{k}(U(\xi))$ then $U$ can be obtained from the 
ODE system

$$
U^{\prime}(\xi)=\frac{1}{\nabla \lambda_{k} \cdot r_{k}} r_{k}(U(\xi))
$$

provided the genuine nonlinearity condition $\nabla \lambda_{k} \cdot r_{k} \neq 0$ holds.

We are interested in the evolution of an initially uniform mixture that is released and allowed to flow down the incline, so we take initial conditions

$$
h(x, 0)=\left\{\begin{array}{ll}
1 & 0<x<1 \\
0 & \text { otherwise }
\end{array}, \quad \phi_{0}(x, 0)=\phi_{\text {mix }}\right.
$$

The choice of initial condition is not restrictive; up to scaling due to the total volume, the long-term behavior of the solution is independent of the shape of $h(x, 0)$. In the settled regime $\left(\phi_{\text {mix }}<\phi_{c}\right)$, the system evolves into a sequence of rarefaction-shock pairs in which the fronts separate as in the Riemann problem; typical solutions for the monoand bi-disperse cases are shown in Figure 7.

\subsection{Long-term behavior: monodisperse}

The theory for the dilute limit of the dynamic model was studied in 13, where an exact solution for the rarefaction can be found, and the particle front was shown to evolve as $x_{p}=\left(C_{p} t\right)^{1 / 3}+T_{0}+O\left(t^{-1 / 3}\right)$ for some constant $T_{0}$. A similar behavior occurs in the high-concentration limit, where one obtains a rarefaction and a singular shock [21] and the exponent $\alpha$ in $x_{f}(t) \sim t^{\alpha}$ is perturbed slightly from $1 / 3$ due to the accumulation of mass at the front. In both cases, there is a single rarefaction containing particles.

Here we derive a similar result for moderate concentrations, obtaining the limiting solution and the leadingorder correction. It will follow from the analysis that the time scale at which the leading-order asymptotics for the particle fronts become dominant is quite large, and so it cannot be compared directly to the current experimental data. In addition, we identify two sub-regimes that produce different asymptotic behavior for the fronts, depending on whether the critical concentration is below or equal to $\phi_{m}$. First, we follow the approach in 21] (for the highconcentration limit) to derive the limiting solution. The key observation from is that the concentration within the first rarefaction approaches the critical concentration $\phi_{c}$ as $t \rightarrow \infty$. It can be shown 21 that if $h(0)=0$ then necessarily $\phi_{0}(0)=\phi_{c}$. Since $x_{p}(t) / t \rightarrow 0$ as $t \rightarrow \infty$, it must be that $\phi_{0} \rightarrow \phi_{c}$ uniformly for $x \in\left[0, x_{p}(t)\right]$ as $t \rightarrow \infty$. This suggests that we should look for a solution to (17) with constant concentration, and then study the linearization of $\phi_{0}$ about $\phi_{c}$ to obtain the next-order correction.

To define this solution, let $\xi=x / t$ and let $\lambda(\phi)$ be the largest eigenvalue for the Jacobian of (17) (note that $\lambda$ is independent of $h$ ). The system only admits a rarefaction $(h(\xi), h(\xi) \phi(\xi))$ with $\phi_{0}$ constant if $\phi_{0}=\phi_{c}$, so the limiting value of $\phi$ is independent of initial conditions - it is only a function of the system parameters. This solution is

$$
h(x, t)= \begin{cases}0 & \xi<0 \\ \sqrt{\xi /\left(3 f\left(\phi_{c}\right)\right)} & 0<\xi<x_{p}(t) / t \\ \sqrt{\xi} & x_{p} / t<\xi<x_{f} / t \\ 0 & \xi>x_{f} / t\end{cases}
$$

Setting $r:=\phi_{\operatorname{mix}} / \phi_{c}$, the front positions are $x_{p}=\left(C_{p} t\right)^{1 / 3}$ and $x_{f}=\left(C_{f} t\right)^{1 / 3}$ with constants

$$
C_{p}=\frac{9}{4}\left(3 r^{2} f\left(\phi_{c}\right)\right), \quad C_{f}=\frac{9}{4}\left(1-r\left(1-\sqrt{3 f\left(\phi_{c}\right)}\right)\right)^{2}
$$

which can be obtained from particle and fluid conservation

$$
\begin{aligned}
\phi_{\text {mix }} & =t \int_{0}^{x_{p}(t) / t} h(\xi) \phi_{0}(\xi) \mathrm{d} \xi \\
1 & =t \int_{0}^{x_{p}(t) / t} h(\xi) \mathrm{d} \xi+t \int_{x_{p}(t) / t}^{x_{f}(t) / t} \sqrt{\xi} \mathrm{d} \xi .
\end{aligned}
$$

Plots of the limiting solution against numerical simulations are shown in Figure 8. For physical context, in the model of 13 with typical experimental parameters, 1500 units of non-dimensional time corresponds to about 10 minutes, by which point the particle fronts have traveled on the order of one meter along the incline. If the long-time behavior with $\phi_{0} \rightarrow \phi_{c}$ is correct, then the ratio of the fronts converges to a positive constant:

$$
\lim _{t \rightarrow \infty} \frac{x_{p}(t)}{x_{f}(t)}=\left(3 r^{2} f\left(\phi_{c}\right)\right)^{\frac{1}{3}}\left(1-r\left(1-\sqrt{3 f\left(\phi_{c}\right)}\right)\right)^{\frac{2}{3}} .
$$

In the dilute limit, the ratio 23 reduces to the result derived by Murisic et. al. 13] with $\phi_{c}=B / 2$. The behavior of the rarefaction near $\phi_{c}$ can then be obtained by solving for the concentration $\phi_{0}$ in the region containing particles. The ODE system 18 for the rarefaction is (with $\lambda^{\prime}=\frac{d \lambda}{d \phi_{0}}$ )

$$
\begin{aligned}
h^{\prime}(\xi) & =\frac{1}{h} \frac{1}{2 \lambda+\frac{\lambda^{\prime}}{f^{\prime}}(\lambda-3 f)}, \\
\left(h \phi_{0}\right)^{\prime}(\xi) & =\frac{1}{h} \frac{\lambda-3 f+\phi_{0} f^{\prime}}{2 \lambda f^{\prime}+\lambda^{\prime}(\lambda-3 f)} .
\end{aligned}
$$

It follows from the above and the condition $\xi=h^{2} \lambda$ that

$$
\phi^{\prime}=\frac{1}{\xi} \frac{\lambda(\lambda-3 f)}{2 \lambda f^{\prime}+\lambda^{\prime}(\lambda-3 f)} .
$$

This is a single ODE for $\phi_{0}$; the appropriate boundary conditions are $\phi_{0}(0)=\phi_{c}$ as discussed earlier and $\phi_{0}(1)=$ $\phi_{\text {mix }}$. The entropy condition ensures that the denominator of (24) is single-signed; we can check by computing the eigenvalue explicitly that $\lambda-3 f<0$ for $\phi_{0}<\phi_{c}$ and $\lambda-3 f>0$ for $\phi_{0}>\phi_{c}$, so it follows that $\phi_{c}$ is the unique attracting fixed point as $\xi \rightarrow 0$ as expected. We can now linearize 24) about $\phi_{c}$ and obtain an expression for $h=$

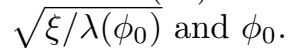


There are two cases to consider here, since the fixed point $\phi_{c}$ may equal $\phi_{\max }$. The linearization depends on the behavior of $R(\phi)$ as defined in (11), which satisfies $R\left(\phi_{c}\right)=$ 0 . If $\phi_{c}<\phi_{m}$ we assume in addition that $R^{\prime}\left(\phi_{c}\right)<0$ with the inequality being strict; which should hold for any reasonable model of the fluxes. However, $R^{\prime}\left(\phi_{m}\right)=0$, a consequence of the viscosity $\mu(\phi)$ becoming infinite as $\phi \rightarrow \phi_{m}$. For the viscosity relation used here, it holds that $R^{\prime}\left(\phi_{m}\right)=R^{\prime \prime}\left(\phi_{m}\right)=0$ [20]. Thus there are two cases to consider for the linearization, depending on whether the degeneracy is present.

Note that for the incline problem, the critical concentration increases with angle, so we can define $\alpha^{*}$ to be the angle for which $\phi_{c}=\phi_{m}$ (if it exists). In the equilibrium model we can find this exactly:

$$
\cot \alpha^{*}=\frac{9 \phi_{m} K_{c}}{2 \rho_{s}\left(1-\phi_{m}\right)}\left(1+\rho_{s} \phi_{m}\right)
$$

For typical experimental parameters, $\alpha^{*} \approx 15.4 \mathrm{deg}$, which is well within the range of angles for which the model assumptions are expected to be valid.

\section{Case $1\left(\phi_{c}<\phi_{m}\right)$ :}

The exact expression for the scaled eigenvalue $\lambda$ is

$$
\lambda=3 f-\frac{R^{\prime}+2 f}{2}+\frac{1}{2} \sqrt{\left(R^{\prime}+2 f\right)^{2}-12 f^{\prime} R} .
$$

Note that $\lambda\left(\phi_{c}\right)=3 f\left(\phi_{c}\right)$ and $\lambda>3 f$ precisely when $\phi>\phi_{c}$. With $p=\phi_{0}-\phi_{c}$,

$$
\lambda-3 f=-3 f^{\prime}\left(\phi_{c}\right) \frac{R^{\prime}\left(\phi_{c}\right)}{R^{\prime}\left(\phi_{c}\right)+2 f\left(\phi_{c}\right)} p+O\left(p^{2}\right) .
$$

The linearization of (24) about $\phi_{c}$ is therefore

$$
p^{\prime}=\eta \frac{p}{\xi}+O\left(p^{2}\right), \quad \eta:=-\frac{3}{2} \frac{R^{\prime}\left(\phi_{c}\right)}{R^{\prime}\left(\phi_{c}\right)+2 f\left(\phi_{c}\right)}
$$

so $p \sim \phi_{c}+K_{p} \xi^{\eta}$ to leading order for some constant $K_{p}$. The prefactor $K_{p}$ is determined by the constraint $\phi_{0}(1)=\phi_{\text {mix }}$, but is difficult to obtain explicitly since the approximation is only valid as $\xi \rightarrow 0$. We do have, however, the simple approximation $K_{p} \approx \phi_{\mathrm{mix}}-\phi_{c}$.

In the equilibrium model it is straightforward to compute the exponent $\eta$ by exploiting the fact that the concentration profile $\phi(x, z, t)$ from 10 is constant in $z$ at the critical concentration; the result is

$$
\eta=-\frac{\beta}{2(\beta+2)}, \quad \beta:=\frac{1+2 \rho_{s} \phi_{c}+B \rho}{\left(1+c_{1} \frac{\phi_{m}}{\phi_{m}-\phi_{c}}\right)\left(1+\rho_{s} \phi_{c}\right)} .
$$

Note that $\eta \rightarrow 0$ as $\alpha \rightarrow \alpha^{*}$ from above since $\phi_{c} \rightarrow \phi_{m}$, so the particle concentration will tend to converge more slowly as $\phi_{c}$ increases.
Case $2\left(\phi_{c}=\phi_{m}\right)$ :

Assume that $R^{\prime}\left(\phi_{m}\right)=R^{\prime \prime}\left(\phi_{m}\right)=0$. and set $p=\phi-$ $\phi_{m}$. Further assume the expansions

$$
R(\phi)=\frac{a}{6} p^{3}+O\left(p^{4}\right), \quad f^{\prime}(\phi)=b p+O\left(p^{2}\right) .
$$

Explicit formulas for the constants $a, b$ are derived in [20]. Expanding the expression 25 for $\lambda$ gives

$$
\begin{aligned}
\lambda & =3 f-\frac{R^{\prime}+2 f}{2}+\frac{1}{2} \sqrt{\left(R^{\prime}+2 f\right)^{2}-12 f^{\prime} R} \\
& =\left(\frac{3 b}{2}-a / 2\right) p^{2}+O\left(p^{3}\right) .
\end{aligned}
$$

The ODE for $\phi$, linearized now about $\phi_{m}$, is similar to Case 1 with a different exponent:

$$
p^{\prime}=\eta \frac{p}{\xi}, \quad \eta=-\frac{a}{4 b-2 a} .
$$

so $\phi \sim \phi_{m}-K_{p} \xi^{\eta}$. Substituting the values of $a$ and $b$ from [20] allows the exponent to be written in a form analogous to Case 1 . The result is

$$
\eta=-\frac{\beta}{2(\beta+2)}, \quad \beta:=\frac{\phi_{m}\left(1+\rho_{s} \phi_{m}\right)+B\left(\phi_{m}-1\right)}{c_{1} \phi_{m}\left(1+\rho_{s} \phi_{m}\right)}
$$

The exponent $\eta$ for both cases is shown in Figure 9; note that there is indeed an angle $\alpha^{*} \approx 15.4 \mathrm{deg}$ such that $\phi_{c}=$ $\phi_{m}$ for $\alpha<\alpha^{*}$ according to the model.

\subsection{Front positions: monodisperse}

This can be used to obtain the desired asymptotic behavior for the front positions. Again, we consider the two cases separately.

Case $1\left(\phi_{c}<\phi_{m}\right)$ :

Expanding $h^{2} \lambda=\xi$ to first-order in $p$ using (26) yields

$$
h(\xi)=\frac{\xi^{1 / 2}}{\sqrt{\lambda\left(\phi_{c}\right)}}-\frac{K_{p} \lambda^{\prime}\left(\phi_{c}\right)}{2 \lambda\left(\phi_{c}\right)^{3 / 2}} \xi^{1 / 2+\eta}+O\left(p^{2}\right) .
$$

Conservation of particles (21), up to the second term, is then (after some simplification)

$$
\begin{gathered}
\phi_{\text {mix }}=t \int_{0}^{x_{p}(t) / t} \frac{\phi_{c}}{\sqrt{\lambda\left(\phi_{c}\right)}} \xi^{1 / 2}+K_{1} \xi^{1 / 2+\eta} \mathrm{d} \xi+\cdots, \\
K_{1}=\frac{K_{p}}{\sqrt{\lambda\left(\phi_{c}\right)}}\left(\frac{3 f\left(\phi_{c}\right)-g^{\prime}\left(\phi_{c}\right)}{R^{\prime}\left(\phi_{c}\right)+2 f\left(\phi_{c}\right)}\right) .
\end{gathered}
$$

This yields the leading order term, which is exactly the limiting solution 20], and first correction:

$x_{p}(t) \sim\left(C_{p} t\right)^{\frac{1}{3}}+C_{1, p} t^{\frac{1-2 \eta}{3}}, \quad C_{1, p}:=-K_{1} \frac{\sqrt{3 f\left(\phi_{c}\right)} C_{p}^{(1+\eta) / 3}}{\phi_{c}(\eta+3 / 2)}$.

The leading order expression for the fluid front can then be determined from fluid conservation 22 to be $x_{f}(t) \sim$ $\left(C_{f} t\right)^{1 / 3}+O\left(t^{(1-2 \eta) / 3}\right)$, and the ratio of the fronts is then

$$
x_{p}(t) / x_{f}(t) \sim\left(C_{p} / C_{f}\right)^{1 / 3}+O\left(t^{-2 \eta / 3}\right) .
$$


Note that the factor $\eta$ does not affect the leading order behavior, but controls the time at which the limiting solution becomes the dominant term; the expansion is in powers of $t^{-2 \eta / 3}$. Since $\eta$ is increasing with $\alpha$ (plotted in Figure 9, left panel), as the inclination angle is decreased, the correction term becomes larger, even approaching $\sim t^{1 / 3}$ as $\alpha$ approaches the angle for which $\phi_{c}=\phi_{m}$. This long time scale for the dominance of the leading order asymptotics is illustrated in Figure 10; the estimate for the front ratios is poor for small angles even up to relatively large times.

Case $2\left(\phi_{c}=\phi_{m}\right)$ :

Here the linearization of $h$ about $\phi_{m}$ takes a different form because the denominator of the rarefaction ODE for $h$ vanishes at $\phi=\phi_{m}$ :

$$
h=\frac{\sqrt{\xi}}{\sqrt{\lambda}} \sim \frac{1}{\left|K_{p}\right| \sqrt{3 b / 2-a / 2}} \xi^{1 / 2-\eta} .
$$

Note that unlike the first case, $h$ is not asymptotic to $\sqrt{\xi}$ as $\xi \rightarrow 0$. As a consequence, 21 suggests that the particle front to leading order is

$$
x_{p}(t) \sim\left(C_{p}^{\prime} t\right)^{\gamma}+\cdots \quad \gamma:=\frac{1-2 \eta}{3-2 \eta}
$$

for some constant $C_{p}^{\prime}$. In contrast, the rarefaction for the clear fluid is given exactly by $\sqrt{\xi}$, and so volume conservation 22 , implies that $x_{f}(t) \sim\left(C_{f} t\right)^{1 / 3}$ to leading order for $C_{f}=\frac{9}{4}\left(1-\phi_{\text {mix }} / \phi_{m}\right)^{2}$. Thus $x_{p}$ grows asymptotically slower than $x_{f}(t)$ (since $\eta>0$ ), so $x_{p} / x_{f} \rightarrow 0$ as $t \rightarrow \infty$ in contrast to the $\phi_{c}<\phi_{m}$ case or the dilute limit. In the ridged regime, the decrease in the asymptotic front speed to $x_{p}(t) \sim t^{\gamma}$ occurs due to mass accumulation at the (singular) shock [21]. Here, the deviation is due to accumulation of particles to the maximum packing fraction in the back of the fluid rather than the front, and the leading clear fluid front is unaffected. This establishes two qualitatively different long-term behaviors - for large angles $\left(\phi_{c}<\phi_{m}\right)$, the ratio $x_{p}(t) / x_{f}(t)$ approaches a constant as $t \rightarrow \infty$ while for small angles $\left(\phi_{c}>\phi_{m}\right)$ the particle front lags arbitrarily far behind the fluid front as the concentration in the particle-laden region approaches $\phi_{m}$. However, because the rate of convergence is so slow, it may be difficult to verify this distinction between the two cases in an experiment.

It is worthing noting that the leading order correction is only valid when $0<\eta<1 / 2$. As is evident from Figure 9 , there is an inclination angle $\alpha_{0}$ such that the above estimate is not valid for $\alpha<\alpha_{0}$ where $\alpha_{0} \approx 7 \mathrm{deg}$ is quite small. Due to the dominance of settling at small angles and the slow speed of propagation, angles in this small range have not been considered in previous works; it may be of limited interest because the applicability of the model is questionable in this regime.

The preceding analysis shows that when the particles and fluid separate, the particle concentration approaches a constant $\phi_{c}$. The distribution of the particles when $\phi_{0}=\phi_{c}$ is uniform (i.e. $\phi=\phi_{c}$ ), and so the particles in the rarefaction initially settle, lag behind the fluid and then become well mixed again as the fluid depletes from this region. If $\phi_{c}<\phi_{m}$, then the particle and fluid fronts both evolve (to leading order) as $t^{1 / 3}$ and their ratio approaches a constant. However, if the inclination angle is small enough then $\phi_{c}=\phi_{m}$ and the particle front evolves as $t^{\gamma}$ with $\gamma<1 / 3$ because the aggregation of particles to the maximum packing fraction impedes the flow.

\subsection{Long-term behavior: bidisperse}

For the bidisperse problem (8), the computations are made much more cumbersome due to the third equation. However, it is easy to extend the monodisperse limiting solution in the settled regimeunder the assumption that it remains appropriate limit for the bidisperse problem (which is suggested by the numerics). Consider initial conditions

$$
\begin{gathered}
h(x, 0)= \begin{cases}1 & 0<x<1 \\
0 & \text { otherwise }\end{cases} \\
\phi_{0}(x, 0)=\phi_{\text {mix }}, \quad X_{0}(x, 0)=X_{\text {mix }}
\end{gathered}
$$

where $\phi_{\text {mix }}$ and $X_{\text {mix }}$ are the initial total and relative concentration of the initially uniform mixture.

Recalling the definition from (11), for each $X$ there is a critical concentration $\phi_{c}(X)$ such that $R\left(\phi_{c}(X), X\right)=0$. For convenience, let $\phi_{c, i}$ be the critical concentration for each species, i.e. values such that $R\left(\phi_{c, 1}, 1\right)=R\left(\phi_{c, 2}, 0\right)=$ 0 . We assume here that the mixture is in the settled regime, i.e. $\phi_{\text {mix }}<\phi_{c, i}$. By an argument analogous to the monodisperse case, it can be shown that a constant concentration rarefaction can only exist if $\phi_{0}=\phi_{c}(X)$ and $X=0$ or $X=1$, which suggests that the long-time behavior has a rarefaction with only heavier particles $\left(\phi_{0}=\phi_{c, 2}\right)$, then a rarefaction with only lighter particles $\left(\phi_{0}=\phi_{c, 1}\right)$, and finally a clear-fluid front. Numerical evidence suggests this is the case (see Figure 11), although convergence of the heavier particle layer to $X=0$ - if at all - is quite slow (even slower, perhaps, than the already slow convergence of $\phi$ to $\phi_{c}$ ). The precise solution is essentially the same as (20) so long as $\phi_{c, i}<\phi_{m}$ for $i=1,2$ :

$$
h(x, t)= \begin{cases}0 & \xi<0 \text { or } x_{f} / t<\xi \\ \sqrt{2 A_{2} \xi} & 0<\xi<x_{2}(t) / t \\ \sqrt{2 A_{1} \xi} & x_{2}(t) / t<\xi<x_{1}(t) / t \\ \sqrt{\xi} & x_{1}(t) / t<\xi<x_{f}(t) / t\end{cases}
$$

where the subscripts $2,1, f$ denote the fronts for the heavier particle, lighter particle and fluid, respectively. The constants are $A_{2}=\frac{1}{6 f\left(\phi_{c 2}, 0\right)}$ and $A_{1}=\frac{1}{6 f\left(\phi_{c 1}, 1\right)}$. The fronts are $x_{i}(t)=\left(C_{i} t\right)^{1 / 3}$ for $i=1,2$ and $x_{f}(t)=\left(C_{f} t\right)^{1 / 3}$ where

$$
C_{1}=\frac{9 \phi_{1}^{2}}{8 A_{1} \phi_{c 1}^{2}}\left(1+\frac{\phi_{c 1}}{\phi_{c 2}} \sqrt{\frac{A_{1}}{A_{2}}} \frac{\left(1-X_{0}\right)}{X_{0}}\right)^{2}, \quad C_{2}=\frac{9 \phi_{2}^{2}}{8 A_{2} \phi_{c 2}^{2}} .
$$


Finally the constant for the fluid is

$$
C_{f}=\frac{9}{4}\left(1-\sum_{i=1}^{2} \frac{\phi_{0, i}}{\phi_{c, i}}\left(1-\left(2 A_{i}\right)^{-1 / 2}\right)\right)^{2} .
$$

Typical solutions are shown in Figure 11. The convergence is not very fast and we need to have $X \rightarrow 0$ (or $X \rightarrow 1$ ) as well as $\phi_{i} \rightarrow \phi_{c, i}$ in the appropriate regions. For $X_{0}<1$, the ratio of the front positions for the particles is

$$
\lim _{t \rightarrow \infty} \frac{x_{p 2}(t)}{x_{p 1}(t)}=\left(1+\frac{X_{0}}{\left(1-X_{0}\right)} \frac{\phi_{c 2}}{\phi_{c 1}} \sqrt{\frac{f\left(\phi_{c 1}, 1\right)}{f\left(\phi_{c 2}, 0\right)}}\right)^{-2 / 3} .
$$

As in the monodisperse case, if $\phi_{c, i}>\phi_{m}$ then the limiting ratio $x_{p, i} / x_{f}$ is expected to be zero.

\section{Experimental comparison}

Finally, we present a qualitative comparison of the predicted front positions to the model to recent experiments. Because it is difficult to reproduce the Riemann initial conditions, experiments testing this model have instead focused on the case of fixed volume initial conditions. Previous investigations have explored the transition between settled and ridged regimes 6]. The experimental data considered here [11] has been collected for angles and concentrations within the settled regime (the rarefaction-singular shock pairs expected in the ridged regime [21] have not been fully explored in experiments). The data tracked is the position of the three fronts obtained from imaging, as the height and concentration profiles are difficult to determine accurately. Due to fingering instabilities (visible in Figure 1, particularly for the leading fluid front), the front positions are estimated using an averaging procedure. In addition, because the experiments begin with a well-mixed suspension, some time is required for equilibrium to be reached. This introduces a parameter $t^{*}$, the time at which the particles reach their equilibrium state. While an estimate can be obtained from a scaling argument, we estimate $t^{*}$ directly, essentially using it as a fitting parameter. The suspension is evolved as well mixed until $t^{*}$, (which is within the range of 20 to 40 seconds) and the equilibrium model is used thereafter. A more thorough understanding of the transient phase would be necessary to obtain both a better comparison, and is of interest for future work.

A series of plots comparing the front positions are shown in Figure 12. Solutions to 8) were computed numerically using an upwind scheme, with the fluxes pre-computed from the equilibrium equations 10. As expected, the three fronts can be observed in the experimental data, and the speed of the fronts is greater for larger angles and concentrations. The fluid front is predicted reasonably well. However, the model appears to somewhat under-predict the particle fronts in most cases, particularly for larger angles where the transient phase is expected to be longer. This may be particularly true for multiple species, as the two types of particles must separate from each other as well as from the fluid. While $t^{*}$ is estimated to be on the order of one minute (so we would hope the model compares well over most of the data), a second transient time might be much later. A suspension that remains partly mixed would behave differently, and for instance might explain the increased speed of the observed front of heavier particles.

\section{Discussion}

We have presented a conservation law model for bidisperse suspensions on an incline, extending the model of Murisic et. al. [13 to multiple species. The Riemann problem exhibits triple shock solutions that describe the evolution of the particle and fluid fronts. The addition of a second species leads to separation between the two types of particles, producing a trailing shock across which the composition of particles changes from mostly heavier to mostly lighter particles while the total concentration is nearly constant. The structure of the Riemann problem is otherwise similar to the single-species case, including the distinction between settled and ridged regimes and the formation of singular shocks [21, 20]. In the case of multiple species, the first shock (for the heavier particles) is always present while the singular shock can only appear for the front of lighter particles.

Motivated by experiments, we have considered constantvolume solutions in the settled regime, which correspond to experiments where a fixed volume of a mixture is released down the incline. In the model, solutions are three rarefaction-shock pairs with mostly heavier particles, then lighter particles, then clear fluid. Preliminary comparison to experimental data shows reasonable qualitative agreement; the general structure of three shocks separating the two particle species and the fluid front is observed in the settled regime, but the front positions are consistently under-predicted by the model. Because the model relies on the assumption that particles are in equilibrium, it cannot be compared to the experimental data at early to moderate times where the suspension may still be partly mixed. A model appropriate to the transient phase may be necessary to better understand the system at early times, and make comparison easier at later times when the model should be applicable. The discrepancies between the current bidisperse model and experiments seem to suggest that the model could be improved; a long transient time due to mixing between the two species is a significant concern.

An adjustment to the equilibrium model by way of different physics would manifest itself as a change in the fluxes for the dynamic equations, producing a similar qualitative behavior. While simple, the general diffusive flux model from which we obtain the fluxes has been successful in comparing to experiments in the monodisperse case. It is not always an accurate reflection of the physics of the problem, particular at concentrations near the maximum 
packing fraction where models based on normal stress differences are more appropriate 14, 15. This is primarily a concern in the ridged regime (which is not the focus here), where the particle concentration approaches $\phi_{m}$ at the front, and in the derived asymptotics for small angles, where this occurs in the back of the particle rarefaction.

In the constant-volume case, we have derived the asymptotic behavior of the front positions for the monodisperse model in the settled regime and applied this to find the leading order behavior for bidisperse suspensions. The front positions are shown to evolve as $t^{1 / 3}$ except at small angles, where the concentration approaches the maximum packing fraction in the rarefaction and ratio of particle to fluid fronts tends to zero. The slow rate of convergence, however presents a significant challenge for experimental comparison, as the time scale at which the asymptotic behavior becomes dominant is large except at large angles ( $\alpha \approx 40 \mathrm{deg}$ ). It would be interesting, though, to see if this transition could be observed in experiments.

\section{Acknowledgements}

We thank Li Wang and Dirk Peschka for helpful conversations, to the reviewers for helpful comments and Sarah Burnett, Andrew Li, Matthew Molinaire and Katherine Varela for their experimental work. This work is funded by NSF grants DMS-1312543 and DMS-1045536.

[1] B. P. Cook, Theory for particle settling and shear-induced migration in thin-film liquid flow, Phys. Rev. E. 78 (2008), 045303.

[2] Robert Dorrell and Andrew J Hogg, Sedimentation of bidisperse suspensions, International Journal of Multiphase Flow 36 (2010), no. 6, 481-490.

[3] Herbert E Huppert, Flow and instability of a viscous current down a slope, Nature 300 (1982), no. 5891, 427-429.

[4] Peter D Lax, Hyperbolic systems of conservation laws and the mathematical theory of shock waves, vol. 11, SIAM, 1973.

[5] S. Lee, Jeffrey Wong, and A. L. Bertozzi, Particle-laden flows of bidensity suspensions, Mathematical Modelling and Numerical Simulation of Oil Pollution Problems, vol. 2, Springer Verlag, 2015.

[6] Sungyon Lee, Aliki Mavromoustaki, Gilberto Urdaneta, Kaiwen Huang, and AndreaL. Bertozzi, Experimental investigation of bidensity slurries on an incline, Granular Matter 16 (2014), no. 2, 269-274 (English).

[7] Sungyon Lee, Yvonne Stokes, and Andrea L Bertozzi, Behavior of a particle-laden flow in a spiral channel, Physics of Fluids (1994-present) 26 (2014), no. 4, 043302.

[8] D. Leighton and A. Acrivos, Shear-induced migration of particles in concentrated suspensions, Journal of Fluid Mechanics 181 (1987), 415.

[9] David Leighton and Andreas Acrivos, Measurement of shearinduced self-diffusion in concentrated suspensions of spheres, Journal of Fluid Mechanics 177 (1987), 109-131.

[10] A Mavromoustaki and AL Bertozzi, Hyperbolic systems of conservation laws in gravity-driven, particle-laden thin-film flows, Journal of Engineering Mathematics (2014), 1-20.

[11] Matthew Molinaire, Andrew Li, Sarah Burnett, and Katherine Varela, Gravity driven particle-laden flow, ftp://ftp.math. ucla.edu/pub/camreport/cam15-11.pdf, 082014.

[12] N. Murisic, J. Ho, V. Hu, P. Latterman, T. Koch, K. Lin, M. Mata, and A. L. Bertozzi, Particle-laden viscous thin-film flows on an incline: experiments compared with an equilibrium theory based on shear-induced migration and particle settling, Physica D 240 (2011), no. 20, 1661-1673.
[13] N. Murisic, B. Pausader, D. Peschka, and A. L. Bertozzi, Dynamics of particle settling and resuspension in viscous liquid films, Journal of Fluid Mechanics 717 (2013), 203-231.

[14] P. R. Nott and J. F. Brady, Pressure-driven flow of suspensions: simulation and theory, J. Fluid Mech. 275 (1994), 157-199.

[15] Prabhu R Nott, Elisabeth Guazzelli, and Olivier Pouliquen, The suspension balance model revisited, Physics of Fluids (1994present) 23 (2011), no. 4, 043304.

[16] Ronald J. Phillips, Robert C. Armstrong, Robert A. Brown, Alan L. Graham, and James R. Abbott, A constitutive equation for concentrated suspensions that accounts for shear-induced particle migration, Physics of Fluids A: Fluid Dynamics 4 (1992), no. 1, 30-40.

[17] Arun Ramachandran and David T Leighton Jr, The effect of gravity on the meniscus accumulation phenomenon in a tube, Journal of Rheology (1978-present) $\mathbf{5 1}$ (2007), no. 5, 1073-1098.

[18] J. M. Revay and J. J. L. Higdon, Numerical simulation of polydisperse sedimentation: equal-sized spheres, Journal of Fluid Mechanics 243 (1992), 15-32.

[19] Anubhav Tripathi and Andreas Acrivos, Viscous resuspension in a bidensity suspension, International Journal of Multiphase Flow 25 (1999), no. 1, 1 - 14.

[20] Li Wang and Andrea L Bertozzi, Shock solutions for high concentration particle-laden thin films, SIAM Journal on Applied Mathematics 74 (2014), no. 2, 322-344.

[21] Li Wang, A Mavromoustaki, AL Bertozzi, G Urdaneta, and K Huang, Rarefaction-singular shock dynamics for conserved volume gravity driven particle-laden thin film, Physics of Fluids (1994-present) 27 (2015), no. 3, 033301. 


\section{Figure Captions}

- Figure 1: Left; schematic for the incline problem. Center and right; images from a typical experiment [11] with $\alpha=20 \mathrm{deg}$, concentrations $\phi_{1}=\phi_{2}=0.15$ of each particle type and at times $t=60 \mathrm{~s}$ and $t=$ $800 \mathrm{~s}$. The dimensions in the image are $0.14 \mathrm{~m} \times$ $0.5 \mathrm{~m}$.

- Figure 2: Fluxes for the system (8) as functions of $\phi$ for varying $X$ at $\alpha=30 \mathrm{deg}$. Arrows indicate direction of increasing $X$, with $X=0,0.25,0.5,0.75,1$. The first row shows the particles fluxes $g_{1}$ lighter) and $g_{2}$ (heavier), and the second row shows the fluid flux and the total particle flux $g$.

- Figure 3: Typical shock solutions for $\left(h, h \phi_{0,1}, h \phi_{0,2}\right)$ with initial states $h_{L}>h_{R}$ and uniform initial concentrations $\phi_{\text {mix }}$ in the domain with $X_{0}=1 / 2$. From left to right: a triple shock solution with $\phi_{0}=0.3$ and intermediate state $h^{*}<h_{L}$, a solution for large concentration $\phi_{\operatorname{mix}}=0.55$ with $h^{*}>h_{L}$ and a shock/singular shock solution due to a small right state $h_{R}$.

- Figure 4: 1-curves for fixed $X_{L}=0.25$ and $\phi_{L}$ varying from 0.3 to 0.55 in the non-conserved variables $\left(h, \phi_{0}, X_{0}\right)$. The curves are nearly parallel to the $X$ axis for $\phi_{L}$ in the settled regime, representing a transition from mostly heavier to mostly lighter particles with little change in height. For large $\phi_{L}$, the change in height can be greater if the intermediate state contains a mixture of both particles.

- Figure 5: Riemann problem for $\phi_{0}$ in the settled regime in the variables $\left(h, \phi_{0}, X_{0}\right)$, in three dimensions (left) and projected onto the $\left(h, \phi_{0}\right)$ plane (right). The plots show the 1-shock curve (black) from the given left state, family of 2-shock curves emanating from it, and 3-shock curve associated with the given right state.

- Figure 6: Riemann problem for $\phi$ in the ridged regime projected onto $(h, X)$ and $(h, \phi)$ for left and right states that produce a triple shock with large intermediate height. For smaller choices of $h_{R}$, the $3-$ curve (green) will not intersect the family of 2 -curves, leading to a singular shock solution, just as in the monodisperse case [20].

- Figure 7: Typical profiles for constant-volume solutions to the mono- and bidisperse system with $\alpha=$ $30 \mathrm{deg}$ and $\phi_{\text {mix }}=0.3$, shown at $t=500$. The initial conditions are a volume of mixture of height and length one.

- Figure 8: Constant-volume solution of the monodisperse problem (17) for $\alpha=20$ (left) and $\alpha=40 \mathrm{deg}$ (right) with $\phi_{\operatorname{mix}}=0.3$ at a moderate time $t=$ 500. The dashed lines are the limiting solution 20 .
The agreement is good for large $\alpha$ but becomes slow to converge as $\alpha$ decreases, in accordance with the asymptotics.

- Figure 9: Plot of the exponent $\eta$ in $\phi_{0}(\xi) \sim \phi_{c}+$ $C \xi^{\eta}$ (left) and growth rate $\gamma$ of the particle front $x_{p}(t) \sim\left(C_{p} t\right)^{\gamma}$ against angle $\alpha$ for the equilibrium model when $\phi_{c}<\phi_{m}$ (solid, from Eq. (26)) and $\phi_{c}=$ $\phi_{m}$ (dashed, from Eq. (28)). The transition occurs at $\alpha^{*} \approx 15.4 \mathrm{deg}$; for $\alpha>\alpha^{*}$, the value of $\gamma$ is $1 / 3$, and for $\alpha<\alpha^{*}$ it is given by Eq. 29.

- Figure 10: Numerically computed front positions (solid) compared to the limiting asymptotics with and without the second term (dot-dashed and dashed, respectively). The left panel shows the particle front, and the right panel shows the ratio of the front positions. The constant $K_{p}$ is used effectively as a fitting parameter; it is taken to be $K_{p}=1$ for $\alpha=40 \mathrm{deg}$ and $K_{p}=0.66$ for $30 \mathrm{deg}$ and $K_{p}=0.4$ for $20 \mathrm{deg}$.

- Figure 11: Numerical solution (solid) and limiting solution (30) (dashed) for the bidisperse problem at $t=500$ with $\phi_{\text {mix }}=0.3$ and $\alpha=30 \mathrm{deg}$ (top) and $\alpha=40 \mathrm{deg}$ (bottom). The right panels show $\phi_{0}$ and $X_{0}$; note that $X_{0} \approx 1$ in the second rarefaction. For very long times, $X \rightarrow 0$ in the first rarefaction, i.e. the particle species separate.

- Figure 12: Front positions for the bidisperse system with fixed volume initial conditions for angles $\alpha=15,20,25 \mathrm{deg}$ (left to right) and settled concentrations $\phi_{\text {mix }}=0.2,0.3$. The solid lines are experimental data and dashed lines are front positions from numerical solutions of the system (8), with the initial time chosen to fit the data. 
Figure 1

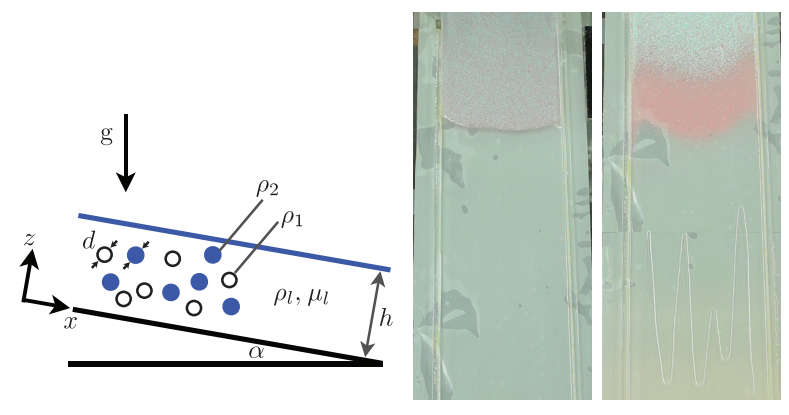


Figure 2
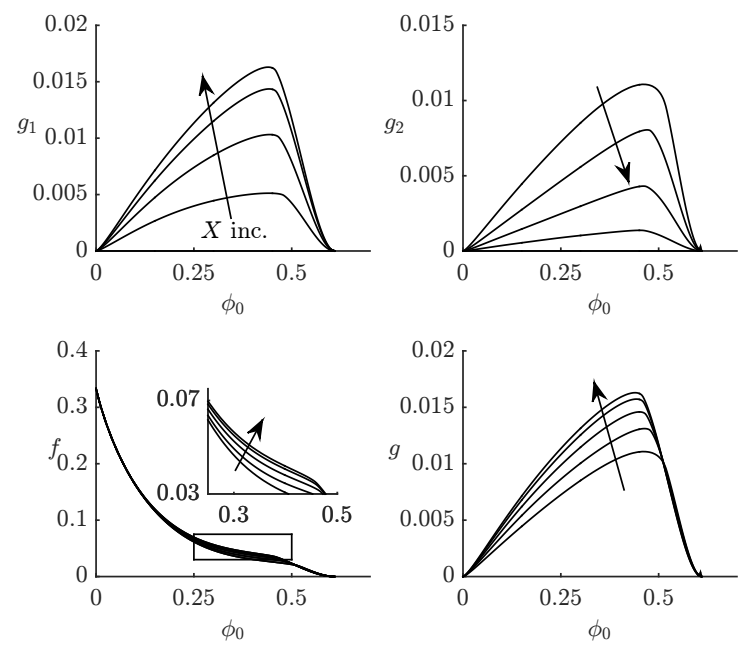
Figure 3
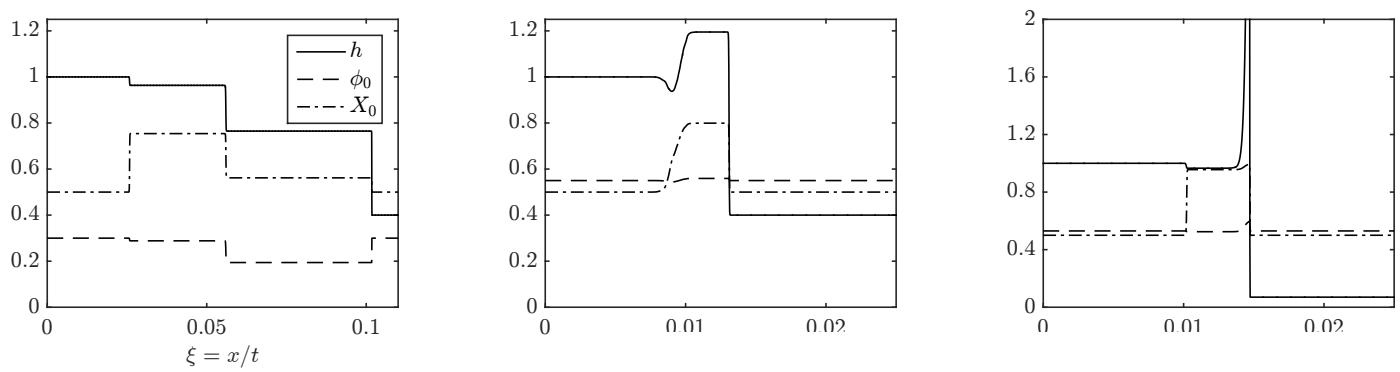
Figure 4

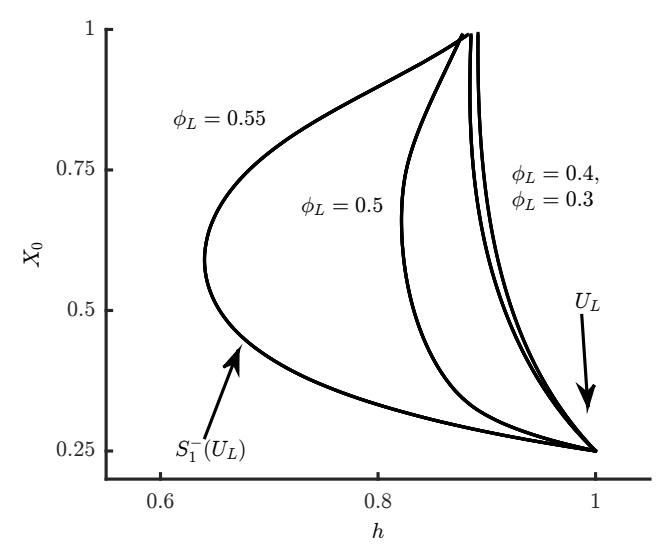


Figure 5
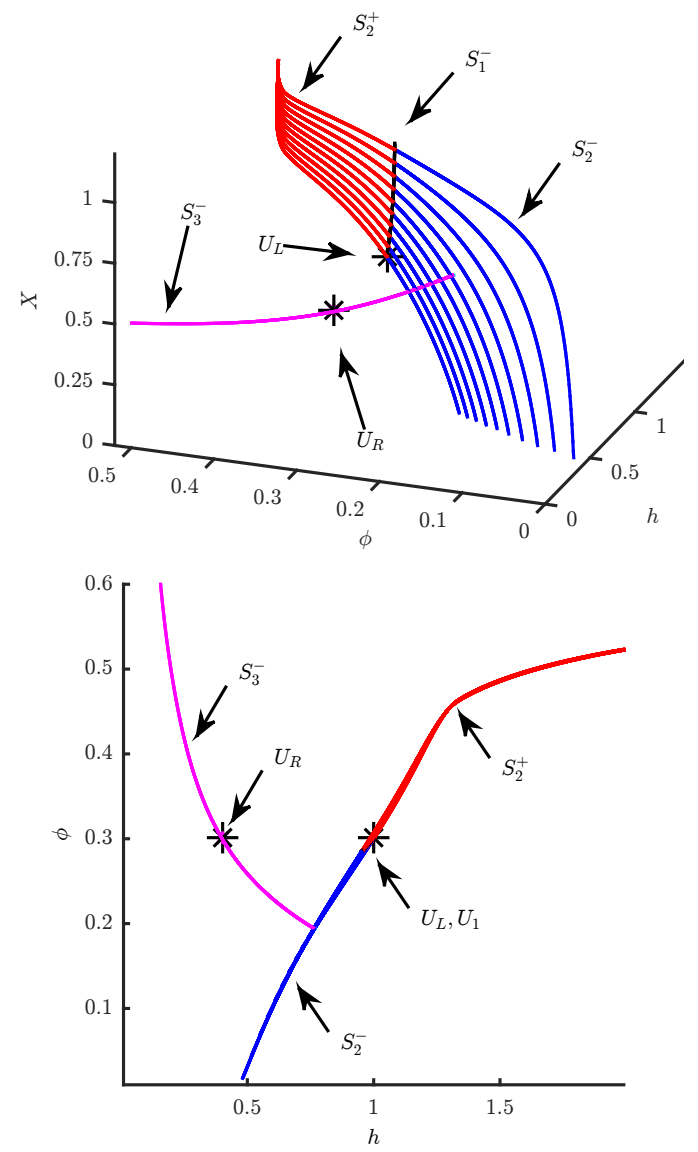
Figure 6
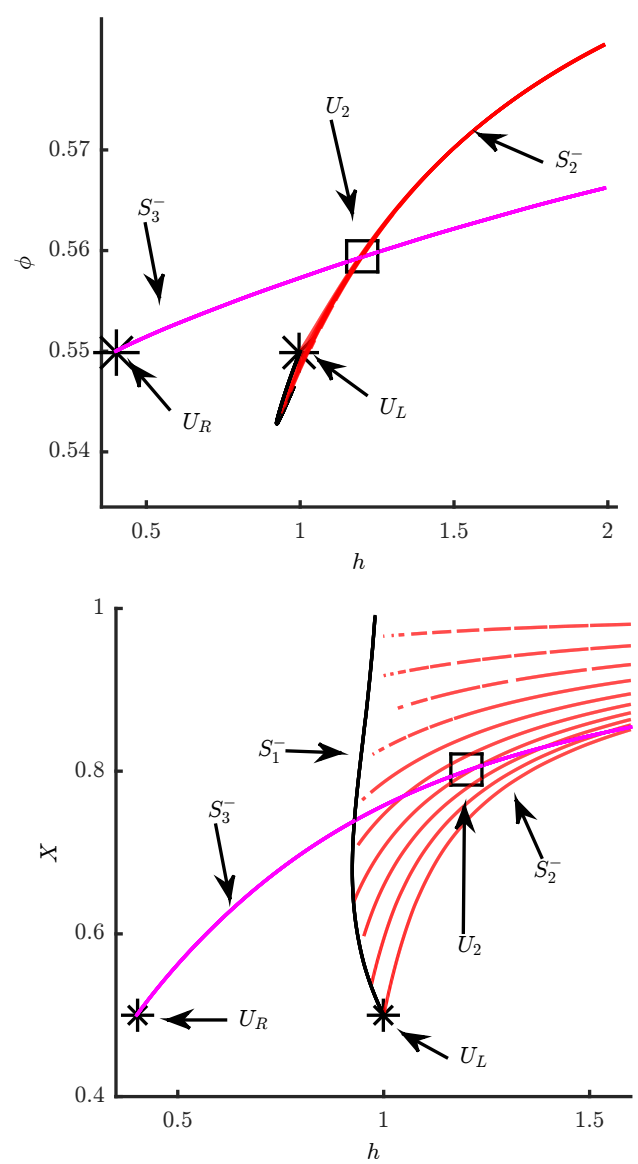


\section{Figure 7}
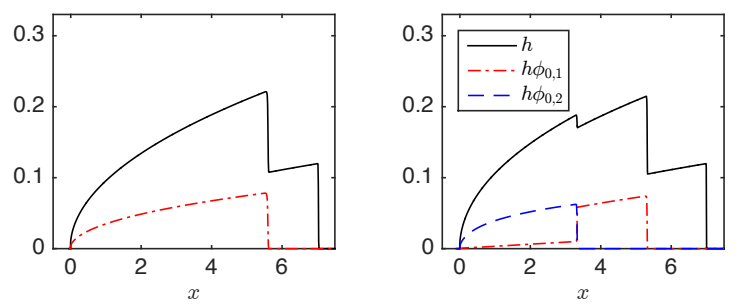
Figure 8
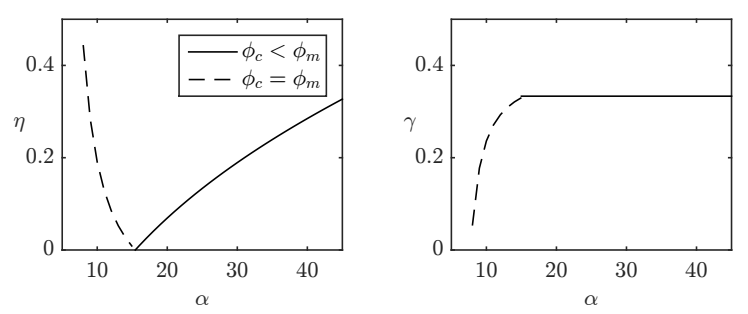
Figure 9
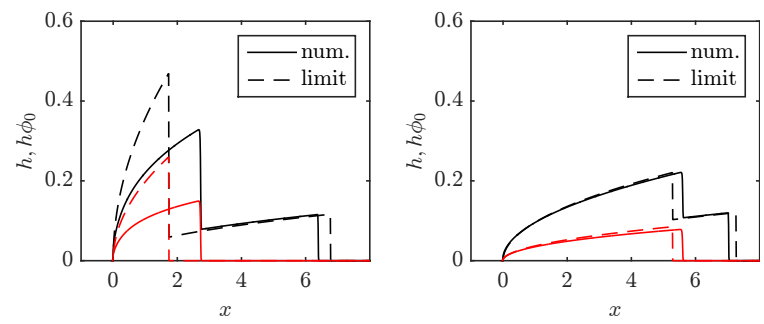
Figure 10
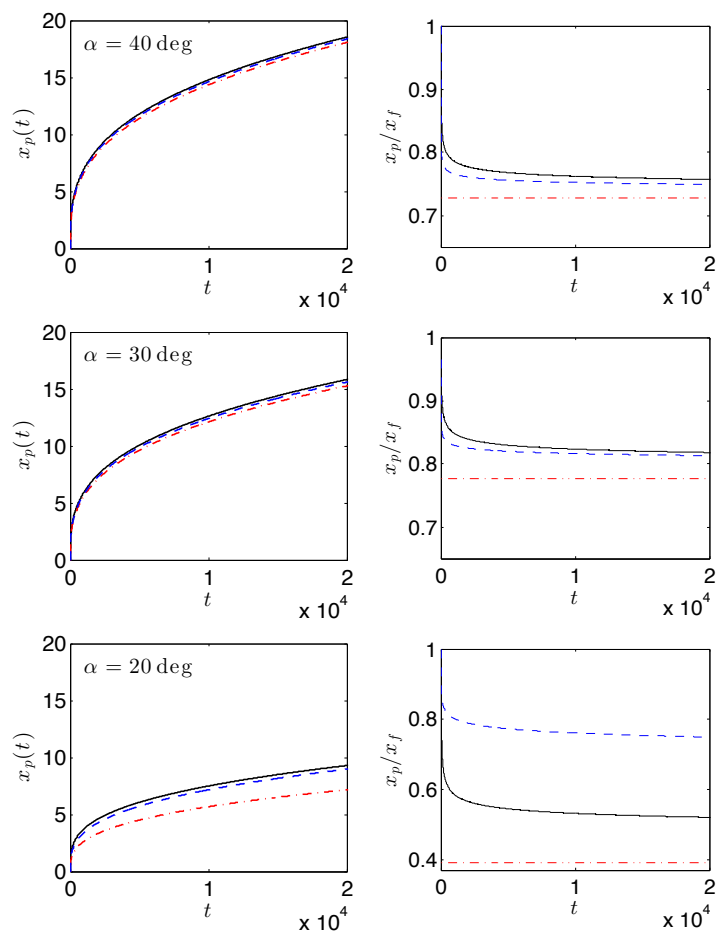
Figure 11
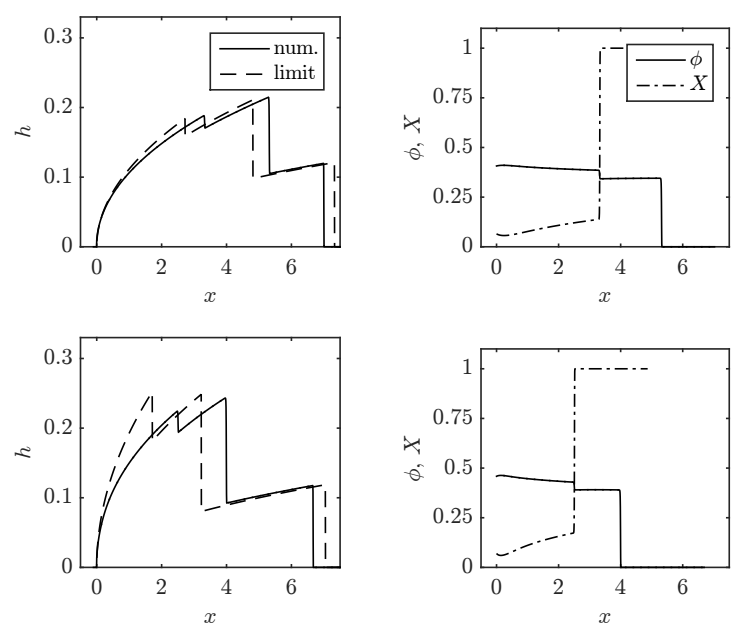
Figure 12
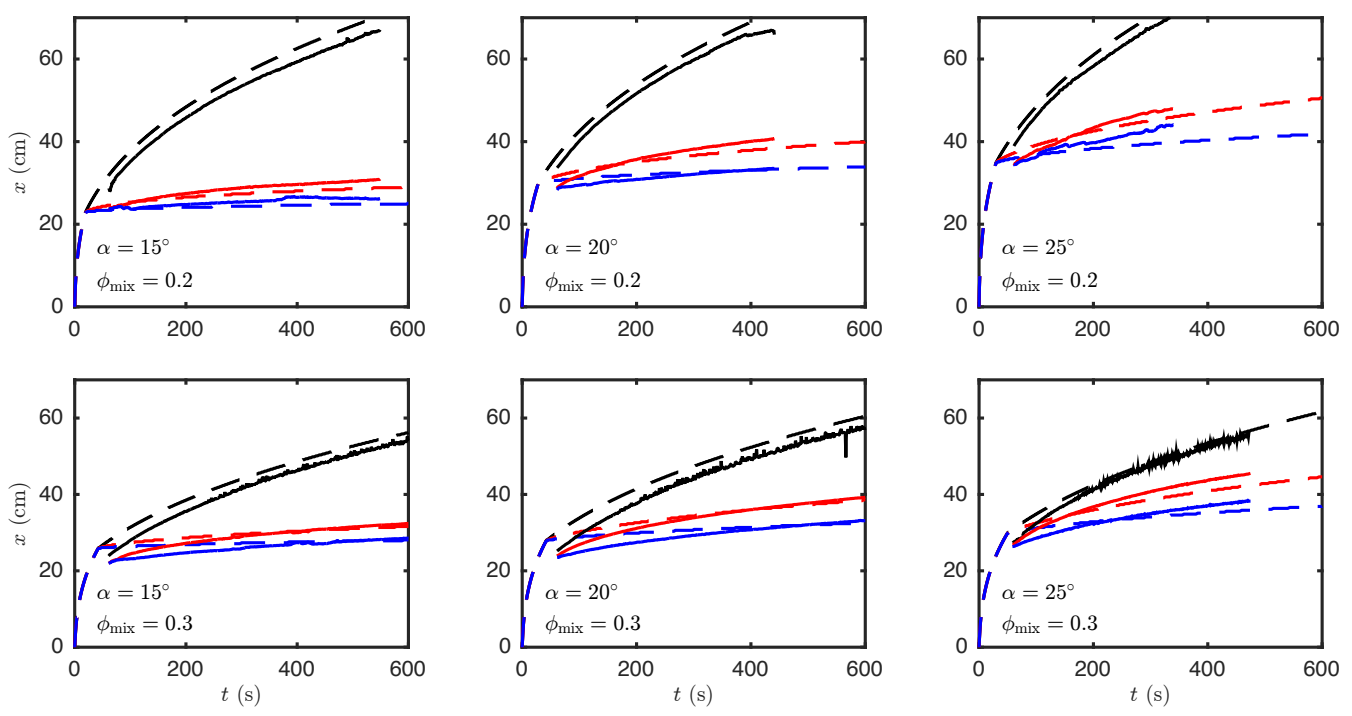